\title{
Effect of aluminium chloride on bond strength of adhesive resins
}

\author{
Keyvan Saati, Haleh Kazemi Yazdi, Naser Valaei, Bahar Bonakdar*, Pedram Khodadadzadeh \\ and Ehsan Seyed Yousefi \\ Islamic Azad University of Medical Sciences-Dental Branch, Tehran, Iran
}

\begin{abstract}
Bond strength of the composite restorations is one of the important factors to achieve success in restoring teeth. This in vitro study evaluated the effect of a hemostatic agent on shear bond strength of a two-step self-etch and a Universal bonding agents. Forty extracted human premolars without any caries, cracks and restorations were selected. After disinfection process, the mesial or distal dentin surfaces were exposed and polished for the same time to gain similar smear layers. They randomly assigned to four groups as follows: $(n=10)$ A: ViscoStat Clear + All-Bond SE / B: ViscoStat Clear + All-Bond Universal / C: All-Bond SE / D: All-Bond Universal. ViscoStat Clear was applied according to manufacturer's orders. Then it was rinsed with constant pressure and the rinsing time was as long as the application time. After 1000 rounds of thermocycling, SBS test was performed. Data were analyzed with 1-way ANOVA.Not significant statistical differences were obtained between control and test groups. Based on the results of this study, there is no statistical differences between control groups and ViscoStat Clear-contaminated groups when using a self-etch or universal bonding agent.
\end{abstract}

\section{KEY WORDS: SELF-ETCHING ADHESIVE, SHEAR BOND STRENGTH, UNIVERSAL BOND, VISCOSTAT CLEAR}

\section{INTRODUCTION}

Bond strength of composite restorations is one of the important factors to achieve success in restoring the teeth. Low bond strength can lead to debonding the restorations. In bonding application process, isolation is an important factor. If the surface is contaminated with

\section{ARTICLE INFORMATION:}

*Corresponding Author: bahar.bnk@gmail.com Received $27^{\text {th }}$ Dec, 2017

Accepted after revision $16^{\text {th }}$ March, 2018

BBRC Print ISSN: 0974-6455

Online ISSN: 2321-4007 CODEN: USA BBRCBA

Thomson Reuters ISI ESC / Clarivate Analytics USA and

- Crossref Indexed Journal

NAAS Journal Score 2017: 4.38 SJIF 2017: 4.196

- A Society of Science and Nature Publication, Bhopal India 2018. All rights reserved.

Online Contents Available at: http//www.bbrc.in/

DOI: $10.21786 / \mathrm{bbrc} / 11.1 / 12$ blood or saliva, bond strength will be reduced. In some cases, blood and saliva control is very difficult especially when the margins of the restorations are under the gum and the operator isn't able to put a rubber dam, (Tuncer et al., 2014). Therefore in these cases, the use of hemostatic agents with the retraction cords is recommended. Sometimes the restoration's margin is not under the 
gum but because of a chronic gingivitis, blood control is difficult. (Tuncer et al., 2014) Another way to control bleeding is the use of lasers and electrosurgery, (Tuncer et al., 2014) but they are not routinely used. The result of application a hemostatic agent might be a reduction in bond strength. To solve this problem, in some studies the rinsing time was increased and some kinds of cleansing methods were selected (Ajami et al., 2013, Chaiyabutr and Kois, 2011), primer application time was increased (Kuphasuk et al., 2007) 6, and also the hemostatic agents and the bonding agents that are more compatible with each other were chosen, (Mohammadi et al., 2012, Fathpour and Khoroushi, 2013.

Some studies showed a SBS reduction when hemostatic agents were used. (Mohammadi et al., 2012, Tuncer et al., 2014, Bernades Kde et al., 2014, Sharafeddin and Farhadpour, 2015) And some of them didn't show any significant differences between test groups and control ones.(Kuphasuk et al., 2007, Fathpour and Khoroushi, 2013, Kimmes et al., 2006) Universal bonding agents or multi-mode one bottle adhesive resins can be used in all of the etch-Ct-rinse, self-etch and selective etch techniques. The technician should select one of the methods according to the cavity and patient's condition.(Hanabusa et al., 2012) In the past studies, there were controversial results about using self-etch adhesive resins with different kinds of hemostatic agents. (Kuphasuk et al., 2007, Arslan et al., 2013).

Nowadays the use of self-etch adhesive resins has increased because there is less technical sensitivity and also procedure time has decreased in this technique, (Arslan et al., 2013, Kuphasuk et al., 2007). Also because of the same penetration depth of resins as the depth of etching, the post-operative sensitivity of patients has decreased, (Mena-Serrano et al., 2013). In this study, the effect of aluminum chloride, as a hemostatic agent, on shear bond strength of the self-etch and the universal bonding agents was evaluated.

\section{MATERIAL AND METHODS}

This study is an experimental, in vitro and a doubleblind one. Forty extracted human premolars without any caries, cracks, and restorations that had approximately the same size were selected. The sample size of each group was obtained via the data from the similar study with sample size formula. (Sharafeddin and Farhadpour, 2015) Samples were kept in distilled water at room temperature. All teeth were cleaned with a rubber cap and the pumice powder. They were stored in $0.2 \%$ Thymol solution for a week then kept in distilled water until the test time. The samples were mounted in self-curing acrylic-resin molds. They were numbered from one to 40 and randomly assigned to four groups. These numbers were kept by someone who did not play a role in the bond strength test. The mesial or distal surface of each tooth was cut by a long, flame bur (Meisinger, Neuss, Germany) with the red round bar until the dentin was exposed. Then the exposed dentin was polished with a 600 grit paper disk for 15 seconds to gain approximately similar smear layer.

In group $\mathrm{A}$, dentine was contaminated with aluminum chloride (ViscoStat Clear, Aluminum chloride 25\%, Ultradent, South Jordan, Utah, USA) according to the manufacturer's instruction.(application time: 5 minutes) It was rinsed with constant pressure and the rinsing time was as long as the application time. Then All-Bond SE ( $6^{\text {th }}$ Generation, self-etching bonding agent, Bisco, Schaumburg, Illinois, USA) was applied in accordance with manufacturer's instruction. Two layers of bonding agent were applied and gently air dried after scrubbing each layer. Then light cured for 20 seconds using a light cure device with an output intensity of $900 \mathrm{mw} / \mathrm{cm}^{2}$ (Demetron LC, Kerr, Orange, California, USA). Forty plastic cylinders were made with internal diameter of two $\mathrm{mm}$ and four $\mathrm{mm}$ height. Ten cylinders were prepared with the following procedure: The composite resin (All-Purpose Body, $\mathrm{A}_{3}$ shade, Bisco, Schaumburg, Illinois, USA) was placed inside the cylinder and fixed with finger on the dentin of the sample. Excess of the composite was removed carefully with a scalpel blade. Then incrementally cured for 40 seconds. The plastic cylinder was cut out.

In group $\mathrm{B}$, dentine was contaminated with aluminum chloride (ViscoStat Clear, Aluminum chloride 25\%, Ultradent, South Jordan, Utah, USA) according to the manufacturer's instruction. And rinsed with constant pressure. The rinsing time was as long as the application time. Then All-Bond Universal (Universal, Bisco, Schaumburg, Illinois,USA) was applied in accordance with manufacturer's instruction. Two layers of bonding agent were applied and gently air dried after scrubbing each layer. Then light cured for 20 seconds using a light cure device with an output intensity of $900 \mathrm{mw} / \mathrm{cm}^{2}$ (Demetron LC, Kerr, Orange, CA, USA). Composite resin (All-Purpose Body, $\mathrm{A}_{3}$ shade, Bisco, Schaumburg, Illinois, USA) was placed and cured on this surface like group A.

In group C (control group), All-Bond SE was used without ViscoStat Clear, and the composite cylinder was applied as the same way in group A.

In group D (control group), All-Bond Universal was used without ViscoStat Clear, and composite resin was placed and cured the same way.

Intensity of the light-curing device was measured (Optilux 501, Demetron, Kerr, Orange, CA, USA) between the procedures.

The specimens were immersed in distilled water after preparation process at room temperature for 24 hours. 
Before the shear bond test, specimens were subjected to 1000-round thermocycling (MP Based, KARA 1000,Tehran, Iran) procedure at $5-55^{\circ} \mathrm{C}$ with a dwell time of 30 seconds and a transfer time of 30 seconds.

In group B and D (universal groups), the self-etch technique was selected to use the universal bonding agent.

Shear bond strength test was performed with a universal testing machine (Zwick/Roell, Z020, Ulm ,Germany) at a crosshead speed of $0.5 \mathrm{~mm} / \mathrm{min}$. Data were analyzed by 1-way ANOVA.

Ethical clearance registration number at Islamic Azad University of Medical Sciences-Dental Branch, Tehran, Iran is "IR.IAU.DENTAL.REC.1396,24". And the ethical ID at IRCT (Iranian Registry of Clinical Trials) is "IRCT2017101136711N1".

This study has been conducted in full accordance with Iranian Registry of Clinical Trials. In our country, ethical issues are reviewed by this center. And the study has been independently reviewed and approved by this ethical board. (It is a publicly accessible database)

\section{RESULTS AND DISCUSSION}

In this study shear bond strength of the composite resin cured to the dentin of the 40 extracted human premolars that were contaminated or uncontaminated with aluminum chloride, were evaluated. Mean shear bond strength values \pm standard deviations of the groups are in Table 1. One-way ANOVA revealed not significant differences in shear bond strength values among selfetch groups and universal groups. Group C (All-Bond SE without the hemostatic agent) indicated the most SBS value $(58.44 \pm 24.07)$ and group B (All-Bond Universal + hemostatic agent) showed the least SBS value. (41.73 \pm 25.78 ) Also, there were no statistically significant differences among four groups. $(\mathrm{P}<0.2)$ The shear bond strength of the both types of bonding agents has not been statistically affected by using the hemostasis. ( $\mathrm{P}$ $<0.4)$. Figure 1 is indicating the comparison of the mean shear bond strength values.

The results of this study showed that the use of aluminum chloride (ViscoStat Clear), as a hemostatic agent,

Table 1. Shear bond strength (MPa) of the specimens $(\mathrm{n}=10)$

\begin{tabular}{|l|l|l|}
\hline Group & Mean+S.D & C.V \\
\hline A (ViscoStat clear+All-bond SE) & $57.13 \pm 38.63$ & 68 \\
\hline B (ViscoStat clear+All-bond universal) & $41.73 \pm 25.78$ & 62 \\
\hline C (All-bond SE) & $58.44 \pm 24.07$ & 41 \\
\hline D (All-bond universal) & $47.20 \pm 25.53$ & 54 \\
\hline
\end{tabular}

has no significant effect on the shear bond strength of composite resin that is bonded with a self-etch (AllBond SE) or a Universal bonding agent on the dentin. After the completion of the test, the data of the current study were placed in the sample size formula and the test power was $80 \%$, so the sample size was sufficient. Aluminum chloride is a useful substance to control bleeding. It has the least side effects and nowadays it is used routinely in clinical procedures.(Kuphasuk et al., 2007) Due to the fact that it doesn't contain iron, like ferric sulfate, it doesn't cause discoloration.(Tarighi and Khoroushi, 2014) Therefore this substance was selected in this study. Most caries end up in dentin, also many of the restorations that we have to use a homeostasis with retraction cords are class $v$ cavities. In many of these cases, decays have reached the root surface and there is no enamel. As a result, dentin was selected for testing in this study.

In the past studies, a $0.5 \%$ chloramine solution was used to disinfect the teeth.(Ajami et al., 2013, Tuncer et al., 2014, Arslan et al., 2012) In this study, because of the scarcity of chloramine, the alternative substance,Thymol, has been used.(Khoroushi et al., 2016, Trakyali and Oztoprak, 2010)

One of the important issues in this process that affects the result, is the removal of the extra composite from the surrounding composite cylinder, which is carefully done in this study. Also, the depth of the dentin is important. As we move towards the pulp, the diameter of the dentinal tubules increases and the resin tags will then be different, (Lopes et al., 2009). The thickness of the smear layer, especially when using self-etching adhesives, is very important. Because in this method, the individual teeth are not etched, the smear layer is not removed and it is only changed, (Arslan et al., 2012). Since the achievement of the same dentin depth is difficult, in this study, an attempt has been made to test the superficial dentin. Also, to reach the same smear layer, cutting and polishing steps were identical. Certainly, the accuracy of this study is less than clinical studies, and this is a disadvantage. In an in vitro study, all the conditions in the clinic can't be achieved. But for the shear bond strength test and using the universal testing machine, the study must be in vitro and clinical studies are not possible.

According to the previous studies, hemostatic agents have acidic PH in the range of 0.7 to 3. They can remove the smear layer and cause demineralization of the enamel and the dentin, (Fathpour and Khoroushi, 2013). One study showed that using aluminum chloride doesn't have any effects on etch- $\mathrm{t}$-rinse bonding agent's strength. In the study mentioned above, ViscoStat and ViscoStat Plus were used as the hemostatic agents. (Kimmes et al., 2006) The use of this hemostasis 


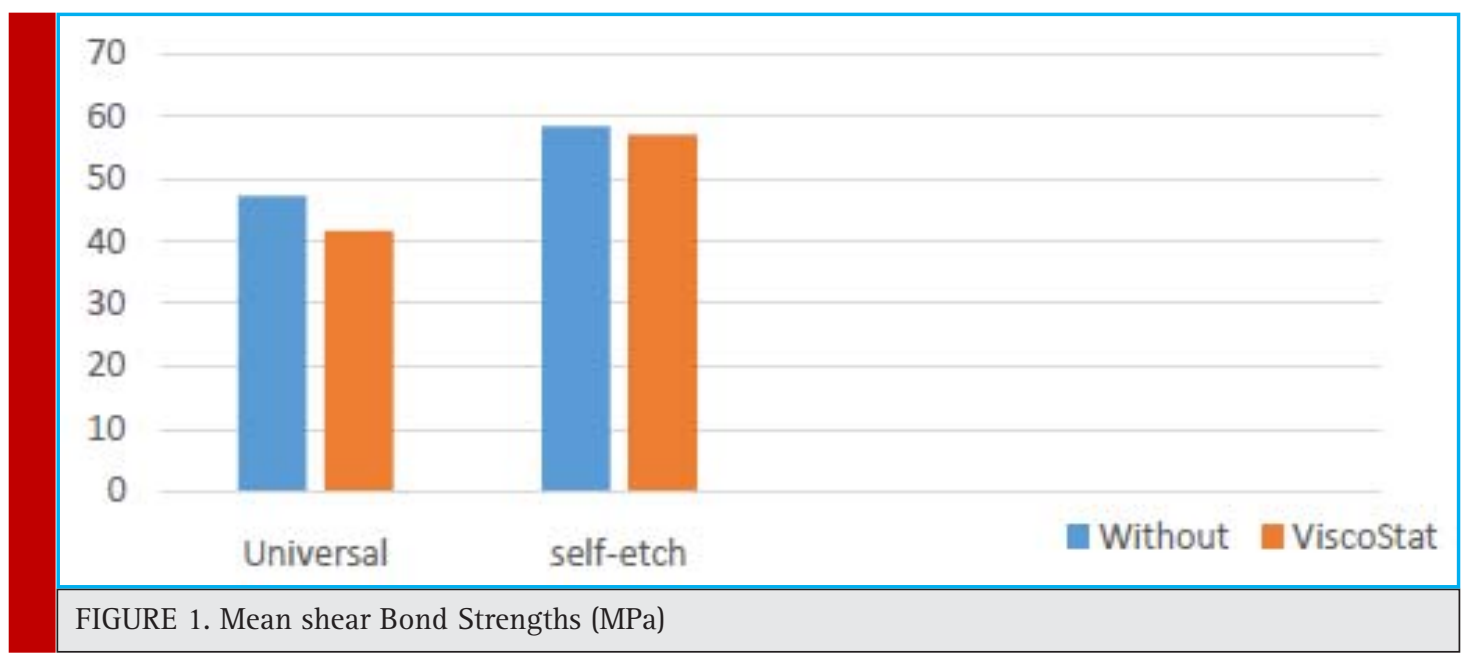

without washing, has a negative effect on bond strength, but if it is removed with plenty of water, it doesn't have any effects on the bond strength, (Kimmes et al., 2006). At least, by washing the hemostasis, the strength will be higher than the situation that the hemostasis is not washed. (0'Keefe et al., 2005). In the etch-Ct-rinse technique, due to the use of phosphoric acid, aluminum does not remain on the surface, (Harnirattisai et al., 2009). Some papers reported a decrease in bond strength even after washing the surface with plenty of water. They had examined the specimens under SEM and reported that the reason for this reduction was the presence of aluminum on the dentine surface, and also the poor acidity of self-etching bonding agents, (Kuphasuk et al., 2007, Mohammadi et al., 2012, Ebrahimi et al., 2013). Therefore, according to these results of the past studies, it is concluded that washing of the surface with plenty of water is very important, especially when using self-etching technique.

Some articles showed that the duration of the application time of the hemostasis and its concentration are important. The longer time it remains on the surface, the more smear layer is removed and more dentinal tubules are open. (Lahoti, 2016) But if it remains on the surface too much, it could cause over-etching. The studies that showed a reduction in the strength of a self-etch bonding agent after using aluminum chloride 25\%, claimed that if the aluminum chloride remains less than 5 minutes on the dentin, it can not remove the smear layer and open the dentinal tubules. On the other hand, because of the poor acidity of self-etching bonding agents, the ability of etching and the penetration of the resin is poor. And bond strength decreases, (Kuphasuk et al., 2007). Based on the results of the previous studies, the property of demineralization of aluminum chloride increases the effect of etching of self-etching bonding agents and prevent the bond strength reduction due to the presence of aluminum, (Kuphasuk et al., 2007, Harnirattisai et al., 2009).

Some studies reported that there are no significant differences between the control groups and ViscoStat Clearcontaminated groups. Also according to an article, some chemical reactions can prevent the bond strength reduction (Khoroushi et al., 2016). After using ViscoStat Clear, the calcium in the hydroxy apatite is replaced by aluminum in $\mathrm{AlCl}_{3}$. This reaction generates $\mathrm{Al}(\mathrm{OH})_{2} \mathrm{H}_{2} \mathrm{PO}_{4}$ which is insoluble. (Ajami et al., 2013, Fathpour and Khoroushi, 2013) As a result, 10-methacryloyloxydecyl dihydrogen phosphate, that is a part of the self-etching adhesives, brings a chemical bond with the remaining minerals in the dentin. Insoluble salts are formed after the use of $\mathrm{AlCl}_{3}$ on the dentin surface. It is probable that due to the formation of a number of chemical bonds, there is no statistically significant difference between ViscoStat Clear- contaminated groups and the control groups, (Khoroushi et al., 2016).

According to one of the studies about self-etching adhesives, the $\mathrm{PH}$ of different kinds of these bonding agents is important. It can cause different results, (Tuncer et al., 2014). Both of the adhesive resins that were used in this study were the self-etch adhesive systems. (All-Bond Universal and All-Bond SE) The PH of All-Bond SE is 2.2 and the $\mathrm{PH}$ of All-Bond Universal is 3.2 that means All-Bond SE is more acidic. By given the mean of the data of this study, it seems that perhaps the use of this substance in the clinic may be somewhat reduced SBS of the composite resin. And it seems that the Universal Bonding adhesive was more affected by aluminum chloride. Perhaps the reason why the Universal Bonding adhesive was more affected by aluminum chloride is its higher $\mathrm{PH}$ and less ability to demineralize the dentine. But since this is an experimental study, it can not clinically prove this issue. According to these controversies mentioned above, more studies are needed. 


\section{CONCLUSION}

Based on the results of this study, there is no statistical differences between control groups and ViscoStat Clearcontaminated groups.

\section{ACKNOWLEDGMENTS}

The proposal related to this article is registered at the Azad University of Medical Sciences - Dental Branch (Tehran, Iran). This study was accomplished in accordance with all the regulations of the local Ethics committee guidelines and policies of Azad University of Medical Sciences- Dental Branch and Iranian Registry of Clinical Trials (IRCT). All the test procedures were performed at Shahid Beheshti University of Medical Sciences (Tehran, Iran). We thank the Dental Research Center, Research Institute of Dental Sciences, Shahid Beheshti University of Medical Sciences, (Tehran 19839 63113, Iran) for doing the tests and their cooperation. The research has not received any funding. The authors declare that they do not have any competing interests or other personal interests of any kind in any products or companies that are presented in this article.

\section{REFERENCES}

Ajami, A. A., Kahnamoii, M. A., Kimyai, S., Oskoee, S. S., Pournaghi-Azar, F., Bahari, M. \&t Firouzmandi, M. 2013. Effect Of Three Different Contamination Removal Methods On Bond Strength of A Self-Etching Adhesive To Dentin Contaminated With An Aluminum Chloride Hemostatic Agent.

Amsler, F., Peutzfeldt, A., Lussi, A. \&t Flury, S. 2017. Long-Term Bond Strength Of Self-Etch Adhesives To Normal And Artificially Eroded Dentin: Effect Of Relative Humidity And Saliva Contamination. J Adhes Dent, 19, 169-176.

Arslan, S., Ertas, H. \& Zorba, Y. 0. 2012. Influence Of Ankaferd Blood Stopper On Shear Bond Strength Of Bonding Systems. Dent Mater J, 31, 226-31.

Arslan, S., Ertas, H. \& Zorba, Y. 0. 2013. Effect Of A PlantBased Hemostatic Agent On Microleakage Of Self-Etching Adhesives. Med Oral Patol Oral Cir Bucal, 18, E124-9.

Bernades Kde, O., Hilgert, L. A., Ribeiro, A. P., Garcia, F. C. \& Pereira, P. N. 2014. The Influence Of Hemostatic Agents On Dentin And Enamel Surfaces And Dental Bonding: A Systematic Review. J Am Dent Assoc, 145, 1120-8.

Chaiyabutr, Y. \& Kois, J. C. 2011. The Effect Of Tooth-Preparation Cleansing Protocol On The Bond Strength Of Self-Adhesive Resin Cement To Dentin Contaminated With A Hemostatic Agent. Oper Dent, 36, 18-26.

Chang, S. W., Cho, B. H., Lim, R. Y., Kyung, S. H., Park, D. S., Oh, T. S. \&t Yoo, H. M. 2010. Effects Of Blood Contamination
On Microtensile Bond Strength To Dentin Of Three Self-Etch Adhesives. Oper Dent, 35, 330-6.

De Carvalho Mendonca, E. C., Vieira, S. N., Kawaguchi, F. A., Powers, J. \& Matos, A. B. 2010. Influence Of Blood Contamination On Bond Strength Of A Self-Etching System. Eur J Dent, 4, 280-6.

Ebrahimi, S. F., Shadman, N. \&t Abrishami, A. 2013. Effect Of Ferric Sulfate Contamination On The Bonding Effectiveness Of Etch-And-Rinse And Self-Etch Adhesives To Superficial Dentin. J Conserv Dent, 16, 126-30.

Fathpour, K. \&t Khoroushi, M. 2013. Effect Of Trichloroacetic Acid Hydrogel On Self-Etch Adhesive Bond Strength To Dental Tissues. J Contemp Dent Pract, 14, 375-80.

Hanabusa, M., Mine, A., Kuboki, T., Momoi, Y., Van Ende, A., Van Meerbeek, B. \&t De Munck, J. 2012. Bonding Effectiveness Of A New 'Multi-Mode' Adhesive To Enamel And Dentine. J Dent, 40, 475-84.

Harnirattisai, C., Kuphasuk, W., Senawongse, P. \&t Tagami, J. 2009. Bond Strengths Of Resin Cements To Astringent-Contaminated Dentin. Oper Dent, 34, 415-22.

Khoroushi, M., Hosseini-Shirazi, M., Farahbod, F. \&t Keshani, F. 2016. Composite Resin Bond Strength To Caries-Affected Dentin Contaminated With 3 Different Hemostatic Agents. Gen Dent, 64, E11-5.

Kimmes, N. S., Olson, T. L., Shaddy, R. S. \&t Latta, M. A. 2006. Effect Of Viscostat And Viscostat Plus On Composite Shear Bond Strength In The Presence And Absence Of Blood. J Adhes Dent, 8, 363-6.

Kuphasuk, W., Harnirattisai, C., Senawongse, P. \&t Tagami, J. 2007. Bond Strengths Of Two Adhesive Systems To Dentin Contaminated With A Hemostatic Agent. Oper Dent, 32, 399405.

Lahoti, K. S. 2016. Effect Of Various Chemical Agents Used In Gingival Retraction Systems On Smear Layer: Scanning Electron Microscope Study. Contemp Clin Dent. 2016/04/ $05 \mathrm{Ed}$.

Lopes, M. B., Sinhoreti, M. A., Gonini Junior, A., Consani, S. Et Mccabe, J. F. 2009. Comparative Study Of Tubular Diameter And Quantity For Human And Bovine Dentin At Different Depths. Braz Dent J, 20, 279-83.

Mena-Serrano, A., Kose, C., De Paula, E. A., Tay, L. Y., Reis, A., Loguercio, A. D. \& Perdigao, J. 2013. A New Universal Simplified Adhesive: 6-Month Clinical Evaluation. J Esthet Restor Dent, 25, 55-69.

Mohammadi, N., Kimyai, S., Bahari, M., Pournaghi-Azar, F. \& Mozafari, A. 2012. Effect Of Aluminum Chloride Hemostatic Agent On Microleakage Of Class V Composite Resin Restorations Bonded With All-In-One Adhesive. Med Oral Patol Oral Cir Bucal, 17, E841-4.

O'keefe, K. L., Pinzon, L. M., Rivera, B. \&t Powers, J. M. 2005. Bond Strength Of Composite To Astringent-Contaminated Dentin Using Self-Etching Adhesives. Am J Dent, 18, 168-72. 
Sharafeddin, F. \&t Farhadpour, H. 2015. Evaluation Of Shear Bond Strength Of Total- And Self-Etching Adhesive Systems After Application Of Chlorhexidine To Dentin Contaminated With A Hemostatic Agent. J Dent (Shiraz), 16, 175-81.

Tarighi, P. \& Khoroushi, M. 2014. A Review On Common Chemical Hemostatic Agents In Restorative Dentistry. Dent Res $\mathrm{J}$ (Isfahan), 11, 423-8.
Trakyali, G. \&t Oztoprak, M. 0. 2010. Plant Extract Ankaferd Blood Stopper Effect On Bond Strength. Angle Orthod, 80, 570-4.

Tuncer, D., Basaran, S., Halacoglu, D. M., Yamanel, K., Celik, C. Et Arhun, N. 2014. Effect Of Haemostatic Agent Application On The Shear Bond Strength Of Contemporary/Multi-Mode Adhesive Systems. Oral Health Dent Manag, 13, 103-6. 